\title{
La estética del entorno y el cognitivismo científico
}

\section{The aesthetics of the environment and scientific cognitivism}

\begin{abstract}
Resumen: Abandonado el estudio de la naturaleza durante casi dos siglos, la estética del entorno entronca hoy con la estética de la naturaleza de Kant y debate sus presupuestos en dos posiciones fundamentales: cognitivismo y no-cognitivismo. En particular, el llamado cognitivismo científico reclama para el juicio estético la mediación imprescindible de conceptos científicos. En el artículo se discute la teoría de Allen Carlson, su principal representante, y se compara con la estética no cognitivista de Malcom Budd.

Palabras clave: cognitivismo, no-cognitivismo, estética del entorno, estética positiva, modelo natural ambiental, conceptos científicos.
\end{abstract}

\author{
MARÍA DEL MAR ROSA MARTÍNEZ
}

\begin{abstract}
For almost two centuries, aesthetitians had completely abandoned the study of the aesthetic of nature. Today's environmental aesthetics links and exposes its interests with the kantian theory of the aesthetic of nature through two main positions: cognitive and non-cognitive views. In particular, scientist cognitivism claims for the essential mediation of scientific concepts in the aesthetic judgement, while anti-cognitivists argue about the need of this requirement and ask for the shared protagonism of other faculties, like imagination. The paper discusses the theory of Allen Carlson, the main representative of the scientific cognitivism, and compares to Malcom Budd's non-cognitive aesthetics.

Key words: cognitivism, non-cognitivism, environmental aesthetics, positive aesthetics, natural-environmental model, scientific concepts.
\end{abstract}

\section{La tradición estética y la estética de la naturaleza}

La estética del entorno es hoy una de las líneas de investigación más fértiles de la estética, tanto en la tradición analítica como en la continental. Las múltiples relaciones que desde la Antigüedad se han dado entre las artes, la filosofía y las ciencias, así como las distintas concepciones sobre el mundo natural provenientes de la cultura entera, forman parte del bagaje histórico de esta rama y constituyen sus antecedentes teóricos y culturales. Entre ellos cabe destacar la influencia de los conceptos de la estética empirista hasta llegar a Kant, la pintura de paisaje desde el siglo siglo XVIII, el protagonismo del arte en detrimento de la naturaleza del siglo XIX y la conciencia científico-política sensible a la protección de los espacios naturales de finales del XIX, que desembocará en el ecologismo del siglo XX.

Fecha de recepción: 13 /11/ 2012. Fecha de aceptación: 14 /01/ 2013.

* Licenciada en Filosofía. Becaria de Formación del Profesorado Universitario. Departamento de Filosofía de la Universidad de Murcia. Correo electrónico: mmar1971@ hotmail.com 
De la combinación problemática de los anteriores factores surgirán los distintos modelos y concepciones de la apreciación estética del entorno que hoy se debaten y estudian.

Yendo más allá de este marco previo, la estética del entorno actual de tradición analítica ha abierto sus focos de atención e interés al incluir como objeto de estudio no sólo a la naturaleza virgen sino también entornos mixtos, aquellos que han sido intervenidos por la acción del hombre, como entornos agrícolas y urbanos, al igual que ha aceptado entre sus tópicos la reflexión sobre la experiencia estética que nos proveen nuestras actividades cotidianas (everyday life), en cualquiera de los entornos mencionados.

Hoy día, en nuestra investigación sobre la experiencia estética de la naturaleza, partimos de una configuración diferente de los elementos tradicionales: la naturaleza y su imagen, la ciencia y sus conceptos, así como de las relaciones que se dan entre ellos. Transcurridos dos siglos de estudios y avances científicos, nuestra representación de la naturaleza ha variado sustancialmente con respecto a la del siglo XVIII que viviera Kant. El mundo de objetos físicos estables de Newton, ha dado paso a nuestro mundo de micro y macro fenómenos abiertos, de procesos y relaciones. Lo que hoy caracteriza a la ciencia es la apertura y el dinamismo de unos fenómenos que no quedan ya al alcance de nuestra intuición. ${ }^{1}$ Tampoco la ciencia nos ofrece hoy una imagen de la naturaleza conformada por un conjunto de objetos, sino de relaciones y procesos que, hasta hace bien poco, nos resultaban inaccesibles en escala y en concepto. No obstante, a pesar del enorme cambio de representación, o quizás también en parte gracias a él, se siguen manteniendo en el centro del debate las consideraciones que Kant propugnara en La Crítica del Juicio sobre la experiencia estética de la naturaleza. A saber: que el juicio estético, en general, es desinteresado; que la experiencia de la belleza natural, frente a la belleza artística, es incondicionada o libre; y que, frente a la experiencia cognitiva de la naturaleza, nuestra experiencia estética de ella es aconceptual. Queda por ver si las modificaciones contemporáneas de nuestra relación con el entorno y las nuevas concepciones de la ciencia han alterado, cómo y en qué medida, los elementos del juicio estético tal y como él los propuso.

\section{Cognitivismo y no-cognitivismo en la estética de la naturaleza}

La estética del entorno se divide en dos líneas de investigación principales cuyas posiciones se conocen como cognitivismo y no-cognitivismo del entorno. Cada una de estas dos líneas defiende que la experiencia estética tiene su centro en el objeto y en el sujeto, respectivamente. Para el cognitivismo, la experiencia estética correcta implica un juicio verdadero sobre la naturaleza. Sin embargo, mientras que para los cognitivistas la apreciación estética de la naturaleza apropiada exige que los objetos de observación estén determinados por conceptos y son éstos los que rigen la apreciación, los no-cognitivistas conviven con el hecho cotidiano de disponer del concepto de algo, pero también con el de carecer de él, y no

1 R. Hepburn: «Contemporary Aesthetics and the Neglect of Natural Beauty», en: Berlant, A., Carlson, A., Berleant (eds): The Aesthetic Appretiation of Natural Environments., Canada, Broadview Press, 2004, p. 44: «el trabajo de las ciencias tiende a incrementar también el desconcierto y la falta de tensión sobre la interpretación estética de la naturaleza. Microscopio y telescopio han añadido vastedad a nuestros datos perceptivos. Las formas de los paisajes ordinarios, normalmente interpretados, se muestran como solamente una sección de entre las muchas e incontables escalas posibles.» [Traducción propia] 
reconocen el papel rector del juicio que les atribuye el cognitivismo. Para el no-cognitivista, la apreciación puede ser correcta también si carecemos de un concepto adecuado para el objeto que apreciamos o si estamos confundidos al respecto. Puesto que, en la apreciación estética, además de conceptos o creencias adecuadas, intervienen de manera relevante otros aspectos cognitivos y sentimentales que dependen del sujeto, como la emoción, la imaginación o la tradición cultural, poniendo todo ello en cuestión el protagonismo de los conceptos.

Podrían considerarse no cognitivistas las teorías que consideran la belleza natural en términos formales ${ }^{2}$, o las que nos proponen juzgar a la naturaleza como si fuera una obra de arte $^{3}$, las que consideran que la experiencia estética no es cognitiva sino que, como la estética del compromiso ${ }^{4}$, la describen en términos de una relación global entre espectador y entorno, o las que la presentan como puramente emocional ${ }^{5}$, o las que consideran la naturaleza como un misterio inabarcable ${ }^{6}$, entre otras.

No todas las posiciones no cognitivistas niegan que la experiencia estética sea aconceptual. La línea no-cognitivista del entorno no se define en su conjunto en contraposición directa a la línea cognitivista, sino que también recoge diversas posiciones respecto a la función que desempeñan los conceptos en el juicio estético. Así, lo que algunas concepciones no cognitivistas rechazan es que los conceptos implicados sean solo de carácter científico, o que la experiencia pueda caracterizarse sólo en función de los conceptos implicados. Por ello, se presentan como ampliaciones de una noción estrecha que concibe el componente cognitivo solo como científico. Ciertos teóricos señalan más a la importancia que poseen conceptos o narraciones de carácter no científico ${ }^{7}$, o de productos de la imaginación ${ }^{8}$, más que a la idea de una ausencia absoluta de conceptos en la apreciación.

\section{El modelo Natural Ambiental de Allen Carlson}

Allen Carlson es uno de los pensadores más destacados dentro de las filas cognitivistas de la estética del entorno actual. Su obra La estética y el entorno. La apreciación de la naturaleza, el arte y la arquitectura ${ }^{9}$ ha marcado el desarrollo central del enfoque cognitivista tanto como el desarrollo del no-cognitivismo, gracias al amplio y rico debate suscitado por sus reflexiones. En este texto, Carlson presenta y defiende un modelo de apreciación estética propio al que da el nombre de Modelo Natural Ambiental. Su concepción de la apreciación se levanta sobre dos ejes principales que parecen requerirse: por un lado, la concepción

2 El paradigma de esta concepción es el planteado por Inmanuel Kant en la Crítica del Juicio. Pero también puede contemplarse como un tipo de apreciación específica, dentro del llamado modelo del paisaje, heredado del pintoresquismo, el cual tomaría las escenas naturales como objetos estéticos análogos a paisajes pictóricos.

3 A. Savile, The test of time: an essay in philosophical aesthetics, Clarendon Press, 1982.

4 A. Berleant, The Aesthetics of Environment, Philadelphia: Temple University Press, 1992.

5 N. Carroll, «On Being Moved by Nature: Between Religion and Natural History», en: Beyond Aesthetics, Cambridge University Press, 2001.

6 S. Goldvlovitch: «Evaluating Nature Aesthetically», Journal of Aesthetics and Art Criticism, 56 (2), 1998 , p.113-125.

7 Th. Heyd, Encountering Nature. Toward an Environmental Culture, Ashgate, 2007.

8 E. Brady, Aesthetics of the Natural Environment, Edinburgh University Press, 2003.

9 A. Carlson, Asthetics and The Environment.The Appretiation of Nature, Art, and Architecture, New York, Routledge, 2000. [Todas las citas de este texto son traducción propia.] 
cognitivista de la apreciación estética y, por otro, sobre su compromiso con la tesis de la «estética positiva». Para quienes la defienden, la naturaleza virgen es, por definición, esencialmente bella y buena. La defensa de esta posición no da cabida a la experiencia estética de la fealdad natural, ya que parte de un estado que podemos denominar como «de belleza natural universal». Esta tesis ha sido históricamente defendida con diferente éxito crítico por teístas, anti-teístas y por otros dialécticos, de corte escéptico.

Carlson considera probado que el conocimiento científico ha desempeñado un rol fundamental en el desarrollo histórico de la apreciación estética de la naturaleza ${ }^{10}$. La apreciación estética del mundo natural creció en contacto con la Ciencia Moderna y ésta fue dotando a la experiencia estética del contexto que necesitaba. Así, aunque la ciencia compartió su capacidad de influencia sobre la experiencia estética con el arte, en el reciente desarrollo de la estética positiva la juega un rol decisivo «si no exclusivo» ${ }^{11}$ en la apreciación. Para Carlson, la mejor justificación de la estética positiva es la que viene sugerida por su propio modelo, esto es, por la apreciación estética de la naturaleza mediada por conceptos científicos. Citando a Rolston III, sucede que los datos (estéticos) de nuestra apreciación son fijados (por medio del conocimiento científico-ecológico) en un contexto mayor, de forma que «vemos belleza ahora donde antes no podíamos verla.» ${ }^{12}$ Por ello, de la mano de las distintas Ciencias la apreciación estética de la naturaleza ha ido encontrando el valor estético positivo que la caracteriza.

\subsection{Cognitivismo de la apreciación y estética positiva}

Ocupando la posición más frontal con respecto a Kant, Carlson defiende que los conceptos científicos poseen una función justificada en el juicio estético de la naturaleza. Carlson levanta su modelo «natural ambiental» de la apreciación estética apoyándose en una crítica de la noción de desinterés. Tradicionalmente, el desinterés estético lo es en dos direcciones: una, que prescinde del contenido de la representación, otra de la existencia del objeto, dando con ello máximo valor a la mera forma. La crítica de Carlson reclama que tanto el contenido de la apreciación, los objetos, como el interés real por la existencia de lo natural, son componentes necesarios para la evaluación estética, en detrimento de la preponderancia de la forma.

Carlson asume las reformulaciones del desinterés kantiano elaboradas por Jerome Stoniltz y Paul Ziff. El primero describe la actitud adecuada del sujeto en el juicio estético como de «atención y simpatía desinteresadas y contemplación de cualquier objeto de conciencia, el que sea, por sí mismo» ${ }^{13}$. Carlson acepta los límites que Stolnitz propone al conocimiento relevante a tener en cuenta por la actitud estética adecuada: no debilitar o destruir la atención que prestemos al objeto, que sea relevante al significado o a la expresividad del objeto y, por último, que mejore la calidad o la significación de la respuesta estética. A la propuesta

10 A. Carlson, 2000, p. 85-87.

11 A. Carlson, 2000, p. 85.

12 A. Carlson, 2000, p. 87: La descripción ecológica encuentra unidad, armonía, interdependencia, estabilidad... los datos iniciales no son negados, solamente redescritos o fijados en un contexto ecológico mayor y en algún lugar encaminan nuestras nociones de armonía, estabilidad, etc...han desplazado también y vemos belleza ahora donde antes no podíamos verla.»

13 A. Carlson, 2000, p. 103: J. Stolnitz, Aesthetics and Philosophy of Art Criticsm, A Critical Introduction, Bostron, Houghton Mifflin, 1960, p. 39. 
de Stoniltz, el hecho de que cualquier objeto puede ser estéticamente interesante y digno de atención, mediado por cierto tipo de conocimiento, Carlson añade la sentencia de Ziff cuando éste matiza que, en realidad, cualquier cosa «lo pide» ${ }^{14} \mathrm{y}$, al hacerlo, nos muestra cómo responder, pues son los objetos en sí mismos los que nos proponen qué ver en ellos a través de distintos «actos de aspección» ${ }^{15}$. Carlson culmina aquí una operación de trasvase del foco de la acción estética del sujeto, en el que todo residía, al objeto, como cuna de propuestas y centro generador de las acciones y respuestas estéticas. Pero, como vemos, su estrategia se desarrolla en base a propuestas que no cancelan por completo la noción de desinterés.

A pesar de que Carlson considera que la apreciación de obras de arte y la apreciación de la naturaleza no son equiparables si comparamos sus objetos, defiende, sin embargo, una concepción unificada de la apreciación estética en base a una analogía en el modo de apreciación estética del arte y la naturaleza. Así, del mismo modo en que la Historia y la Crítica de Arte forman parte del juicio estético de las obras de arte, el conocimiento científico, es decir, la Historia de las Ciencias Naturales (junto con el «sentido común»), es relevante para el juicio estético de la naturaleza. El conocimiento científico nos asiste allí donde el sentido común no llega ${ }^{16}$, cuando no disponemos de una categoría obvia (en el caso de juzgar a una ballena como mamífero, por ejemplo), o en el caso de situaciones estéticas problemáticas o paradójicas (como la apreciación de un tramo de costa artificial, de apariencia natural).

Entrando a debate con la concepción de la verdad y la falsedad de los juicios estéticos sobre arte de Kendall Walton, quien considera que no puede darse el mismo caso con los juicios estéticos sobre la naturaleza, Carlson defiende que sí emitimos juicios estéticos correctos o verdaderos y juicios estéticos incorrectos o falsos acerca de la naturaleza. Según el propio Walton, ciertos juicios estéticos acerca de la naturaleza parecen mostrarse a sí mismos como paradigmáticos, como sucede cuando contemplamos la «majestuosidad de una montaña», o el «carácter sublime de una puesta de sol». Sin embargo, advierte, las condiciones propuestas a la sazón para la evaluación de obras de arte no sirven para la naturaleza puesto que han sido pensadas a partir de un tipo de concepción del arte, de la apreciación, las cualidades y los objetos estéticos, e incluyen cláusulas de origen social. No obstante, en base a la propuesta formulada por Walton, Carlson propone un mecanismo asimilable, una estructura epistémica paralela, aplicable a los juicios de la naturaleza. Las cláusulas relativas a la intención del artista y a la sociología de la recepción de la obra son sustituidas por otras, cuya relevancia justifica socialmente el uso de categorías científicas. De modo que, mientras que las obras de arte y sus categorías pertenecen al reino de la intención y, por tanto, las categorías correctas son relativas al artista, en el caso del juicio estético de la naturaleza, la ciencia ocuparía el lugar del artista al ser ella la encargada de descubrir las categorías con las que conocemos y entendemos la naturaleza. Por lo tanto, al igual que la historiografía crítica nos informa acerca de la tradición artística, la ciencia cumpliría aquí esa función narrativa historiográfica.

14 A. Carlson, 2000, p. 106: P. Ziff, «Anything Viewed,» en: Antiaesthetics, An Appretiation of the Cow with the Subtile Nose, Dordrecht, Reidel, 1984, p. 136: «anything viewed makes demands».

15 Ziff construye esta palabra a partir del cruce de significados de los sustantivos aspecto (aspect) e inspección (inspection).

16 Carlson atribuye vocación preeminentemente científica al lenguaje del «sentido común» asociándole, como continuación natural, las funciones del lenguaje propiamente científico. 


\subsection{Estética positiva}

Carlson considera que su modelo de apreciación provee a la estética positiva de una fundamentación adecuada. En base a él podemos valorar justificadamente, por un lado, cómo la información científica ha sido históricamente la encargada de establecer la relación en la que lo bello y lo bueno se implican en la apreciación estética de la naturaleza. La ciencia moderna, la llamada secularización del mundo moderno, la pintura de paisaje, íntimamente ligada al conocimiento de los avances científicos de su época, y, hoy día, la presencia de las concepciones ecologistas en los pensadores, como Carlson acreedores de la estética positiva, ponen en evidencia la evolución de esta tendencia en el tiempo y la importancia que, así justificada, ha sido atribuida a la ciencia en la apreciación estética de la naturaleza. Para Carlson, la información científica posee relevancia estética, pues ajusta y dirige nuestra mirada, haciendo que prestemos atención a detalles significativos de las cosas que, de otro modo, resultarían estéticamente desapercibidos. A su juicio, el conocimiento científico puede ser enormemente revelador al enfocar nuestra atención, por ejemplo, sobre el tamaño, la forma o el color de una determinada especie de flor alpina, al descubrir en sus rasgos estéticos un mecanismo de adaptación y supervivencia de esa especie en su propio entorno. Así, al igual que Constable afirmaba en 1836 que «pintar es una ciencia y debería ser perseguida como una investigación de las leyes de la naturaleza» ${ }^{17}$, los defensores de la estética positiva caracterizan a «los lenguajes de la naturaleza» como «lenguajes ecológicos» ${ }^{18}$, cuyo valor «tiene que ver con la inteligibilidad de cada uno de los miembros naturales» ${ }^{19}$, atribuyendo que debemos «la intensidad presente del sentimiento por la naturaleza al crecimiento de la ciencia» ${ }^{20}$.

Las representaciones pictóricas de la naturaleza y el pintoresquismo, en general, transmitieron el valor positivo de la naturaleza apoyándose en el conocimiento y la fidelidad científica de los detalles: la diversidad de especies, la grandiosidad de los escenarios, la magnificencia del poder de la naturaleza, la especificidad de seres, formas, colores y funciones naturales, la belleza del diseño vegetal, la sentimentalidad unida a los espacios naturales, los jardines, el tiempo de la naturaleza y el de la arquitectura, el progreso asociado al control del mundo natural, la capacidad de intervención humana en la naturaleza y, al tiempo, su fragilidad. Gran parte de la transmisión histórica del valor estético se habría venido apoyando, así, en la ciencia y sus descubrimientos, en el efecto que el conocimiento científico tiene al hacer de foco sobre las cualidades estéticas de la naturaleza y en la orientación que con ello gana la apreciación estética del objeto, en detrimento de la relevancia que otros atribuyen a la experiencia del sujeto. Por lo tanto, conocer científicamente el mundo natural sería no sólo relevante para su apreciación estética, sino también condición necesaria para su adecuada apreciación. El sen-

17 A. Carlson, 2000, p. 86: K. Constable, de su última conferencia pública, desarrollada en la Royal Insitution of Great Britain, en 1836. Citado por Ronald Rees: «John Constable and the Art of Geography», Geographical Review, vol. 66, 1976, p. 59.

18 A. Carlson, 2000, p. 86: A. Kinnunen: «Luonnonestetijkka» en: A. Kinnunen, Y. Sepanmaa, (eds): Ymparistoestitijkka, Helsinki, Gaudeamus, 1981, p. 49.

19 A. Carlson, 2000, p. 86: H.Rolston, «Can and ought we to follow nature?» en: N. Bunnin y E. P. Tsui-James, (eds.): Environmental Ethics, Oxford: Blackwell Publishing, 2003, p. 23.

20 A. Carlson, 2000, p. 87. 
timiento estético sería entonces hermano o deudor de una percepción del mundo natural que habría estado culturalmente mediada siempre por los ojos de la ciencia moderna.

Carlson reconoce con ello, que la importancia en la apreciación estética de la naturaleza es, en parte, un legado de la concepción estética tradicional con centro en la noción de «desinterés». Siendo crítico, como es, con el énfasis que esa misma tradición pone en el valor estético de la forma, sin embargo, sí admite el valor clasificador formal que las categorías científicas aportan al juicio estético de la naturaleza. Podemos reclamar a Carlson que, en su analogía, hace un uso pragmático, no trascendental, de la justificación del valor del conocimiento científico en la apreciación estética de la naturaleza. Tanto la historia como la crítica de arte son discursos de naturaleza hermenéutica. Por lo tanto, la analogía de Carlson o bien atribuye a la ciencia ese mismo valor de uso, o bien dejaría abierta una brecha en los fundamentos de su corrección.

Por otro lado, vemos cómo, en la ecuación belleza/bondad de su concepción positiva, la justificación de la bondad estética viene aportada por la Ciencia, o por las diversas ciencias en relación que denominamos Ecología, y no por la Estética, debido a que siempre atendemos a lo que la naturaleza es. Carlson quiere salvar este extremo estableciendo un paralelismo entre las cualidades que la naturaleza muestra a la ciencia y las que observamos al apreciar estéticamente la naturaleza, a saber: «orden, regularidad, armonía, equilibrio, tensión, resolución». Afirma que «estas cualidades, que hacen que el mundo nos parezca comprensible, son también aquéllas que nos parecen estéticamente buenas. De modo que, cuando las experimentamos en el mundo natural o experimentamos el mundo natural en término de ellas, lo encontramos estéticamente bueno» ${ }^{21}$. Asimismo añade, «no es sorprendente que sean estas mismas cualidades las que nos parecen estéticamente buenas en arte» ${ }^{22}$.

Carlson fuerza su propia argumentación, llegando a invertir el orden del binomio, cuando sentencia que todo lo dicho es, y lo toma por: «la idea sugerida por aquéllos que afirman que el arte y la ciencia tienen raíces y objetivos similares y por aquéllos que afirman que la ciencia es en parte un refuerzo (endeavor) estético» ${ }^{23}$. Al mismo tiempo reconoce que, más allá de ello, «no está claro por qué debe existir tal conexión entre la bondad estética y la corrección científica», si bien es necesario indicar que «lo que encontramos estéticamente bueno juega un rol importante en lo que nuestra ciencia toma como correcto» ${ }^{24}$.

En este último argumento queda claro en qué consiste la influencia de la estética kantiana para Carlson. La finalidad formal de la naturaleza como principio de todos nuestros juicios, científicos y estéticos, nos obliga a respetar los límites de lo científicamente aceptable. Al fin y al cabo, los objetos bellos de la naturaleza son aquellos en los cuales percibimos una adecuación a nuestra forma de conocer, aún cuando no los conocemos o no los consideramos según nuestro conocimiento de ellos. El principio del juicio o de la finalidad de la naturaleza es el mismo en la experiencia cognitiva de la naturaleza y en la estética. Del mismo modo, se rescata la importancia del desinterés estético, entendido como una actitud distanciada del sujeto respecto del objeto, aunque ahora se enfatice la ausencia de factores subjetivos en la evaluación y el carácter de atención volcada en el objeto frente al sentimiento de la actividad del sujeto.

21 A. Carlson, 2000, p. 93.

22 A. Carlson, 2000, p. 93.

23 A. Carlson, 2000, p. 93.

24 A. Carlson, 2000, p. 93. 


\section{La apreciación estética de la naturaleza de Malcom Budd}

La concepción de la estética de la naturaleza que Budd presenta en su texto The Aesthetic Appretiation of Nature tiene la capacidad de ir respondiendo, desde la estructura de su planteamiento, a la posición de Carlson. Comenzando por la división genérica de la apreciación de la naturaleza entre estética y no estética y siguiendo por la subdivisión de la apreciación estética a partir de su concepción de la respuesta estética, Budd va tejiendo su propuesta, aclarando cuestiones, al tiempo que plantea sus réplicas al cognitivismo de Carlson.

Como digo, Budd distingue entre dos tipos de apreciación estética de la naturaleza. Por un lado, podemos contemplar la naturaleza tomándola como mero objeto, sin atender a su origen, y disfrutarla formalmente. Budd subraya que no existe un requerimiento puramente estético por el cuál algo tenga que ser apreciado necesariamente como la cosa que es. Por lo tanto, no son precisos conceptos científicos específicos para que haya apreciación estética. Ahora bien, si apreciamos la naturaleza, como naturaleza, asociando el objeto a su origen natural, tendremos que definir en qué sentido preciso lo hacemos. En sentido general, la naturaleza posee un significado inclusivo dentro del cual cabe «todo lo que es el caso», el mundo en sentido amplio. Esta primera acepción, no distingue entre naturaleza y artefacto. Para Budd, sin embargo, es necesario que apreciemos estéticamente la naturaleza por lo que ésta es, lo cual requiere, en primer lugar, que no la percibamos como artefacto. No obstante, Budd nos advierte que lo natural no siempre se opone a lo humano ya que, en realidad, el hombre supone un escalón intermedio entre ambas categorías. Por un lado, es un ser natural y como toda otra criatura posee principio de crecimiento intrínseco; por otro, el ser humano es productor o el generador de todo lo que consideramos no natural y sus productos no poseen crecimiento intrínseco. Dentro del espectro de cosas al que nos referimos con la idea de naturaleza (pájaros, amaneceres, tormentas, nidos, graznidos, viento, rocas...), Budd señala que en realidad hacemos mención a tres tipos de cosas: a ítems individuales de una clase, a clases naturales y a fuerzas naturales.

Una última consideración concierne al carácter prístino o no prístino de la naturaleza. Atendemos aquí a tres tipos de escenas: las completamente naturales, aquéllas en las que la naturaleza no muestra intervenciones del medio, otras en las que no hay intervención, pero sí trazas de humanidad (como un huerto, una senda de tierra o actividad ganadera — vacas pastando), y, por último, aquéllas en las que la naturaleza convive con las intervenciones humanas. El segundo tipo en Budd no implicaría que su apreciación debiera ser mixta porque, en ese caso, el factor humano no se opondría al natural, o lo que es lo mismo, el hombre actúa como elemento natural en el entorno, siendo su actividad y sus productos asimilables a los de otros animales, como sucede cuando contemplamos un nido o un panal. Es en ese caso cuando la actividad humana no implica artificio.

Budd presenta dos formas de respuesta a la naturaleza denominadas «concepción externa» $\mathrm{y}$ «concepción interna». La primera implica una lectura débil de la respuesta, ya que parte de la definición de naturaleza como opuesta a lo que no es natural, bien sea arte o artefacto, pudiendo asumir una forma artística y otra no-artística. La segunda implica una lectura fuerte, pues asume que es así en virtud de que algo «es natural» ${ }^{25}$. Esta será una res-

25 M. Budd, The Aesthetic Appretiation of Nature, Oxford, Oxford University Press, 2005, p. 10. [Todas las citas de este texto son traducción propia.] 
puesta a la naturaleza, como naturaleza, lo cuál resulta esencial para el carácter gratificante de nuestra experiencia estética e incluye, como sucede en Kant, la imposibilidad de que el amante de la naturaleza responda ante una réplica imitadora ${ }^{26}$.

Budd considera que la interpretación fuerte puede inducir a cierto escepticismo, ya que alguien podría pensar que es difícil fundamentar el carácter de una respuesta estética en el hecho preciso de que algo sea natural. Pero, a su juicio, es una verdad general que nos deleitamos con los estados, procesos y objetos naturales, y también que nos sentimos conmovidos por ciertos conceptos o descripciones que afectan a nuestra respuesta emocional. Ello da pie a pensar que percibir algo como natural forma parte de la experiencia estética y que nuestra respuesta, en parte, se basa en ello. Para Budd, el hecho de que un objeto o un item sea natural forma parte de su definición y de la fenomenología de su experiencia, y la descripción de un objeto como natural, en sentido interno, infiere que éste no se muestre disponible para definiciones incompatibles.

Buena parte de las diferencias que existen entre cognitivismo y no cognitivismo del entorno tienen su raíz en las diferentes interpretaciones de la noción de desinterés, a pesar de que ambos enfoques parten de una crítica al formalismo que históricamente había lastrado a la noción. Como hemos visto, la crítica cognitivista de Carslon cancela la pasividad distanciada del sujeto y otorga al desinterés el rango de actitud para, acto seguido, reclamar la guía estética del objeto y decantarse por el lado más regular de la información que presenta: los conceptos aportados por las ciencias naturales. Por su parte, la concepción no cognitivista de Malcom Budd va a mantener activas las nociones de contemplación y desinterés, pero no fija una jerarquía de la relevancia, transformada en necesidad, de la información estética. A pesar de que crítica al cognitivismo, Budd no defiende que la apreciación estética carezca de todo conocimiento o contenido. Lo que arguye es que son varios los tipos de experiencia que el objeto reclama y evoca, no sólo en atención a las funciones del entendimiento, sino también según la imaginación. De esta manera, los conceptos según los cuales percibimos los objetos de la naturaleza no son necesariamente científicos, sin que de ello se siga forzosamente un interés del sujeto.

Podemos identificar a Budd como defensor de una noción ampliada del desinterés kantiano. El concepto de desinterés sigue estando en el corazón de la respuesta estética que para Budd consiste en una reacción desinteresada, positiva o negativa, ante las propiedades del item ${ }^{27}$ :

«una respuesta estética es, en la medida en que la respuesta sea dirigida a las propiedades que experimentamos de un item, la naturaleza y disposición de sus elementos o la interrelación entre sus partes o aspectos, e implica una reacción positiva o negativa

26 I. Kant, Crítica del Juicio, Madrid, 1981, Austral. §42: «¿Qué aprecian más los poetas que el canto bello y fascinador del ruiseñor, en un soto solitario, en una tranquila noche de verano, a la dulce luz de la luna? En cambio hay ejemplos de que donde no se ha encontrado ningún cantor semejante, algún alegre hostelero, para contentar a sus huéspedes, venidos a su casa a gozar del aire del campo, los ha engañado escondiendo en un soto a algún compadre burlón, que sabía imitar ese canto como lo produce la naturaleza (con un tuvo o una caña en la boca); pero, conocido el engaño, nadie consentiría en oír largo tiempo esos sonidos, tenidos antes por encantadores, y ocurre lo mismo con cualquier otro pájaro cantor.».

27 Por item, Budd entiende «no sólo objetos físicos o combinaciones de objetos, sino también la actividad o el comportamiento de cosas vivientes o no-vivientes, eventos o procesos de otras clases, meras apariencias, y cualquier otro tipo de cosas susceptible de apreciación estética» (M. Budd, 2005, p. 14). 
hacia el item, no por satisfacer el deseo de existencia o no existencia de algún estado de cosas, en el que el item figura, sino considerado por él mismo (en abstracción de cualquier relación personal que podamos obtener entre sujeto y objeto), de manera que lo que gobierna la respuesta es si el objeto, intrínsecamente, gratifica o displace a la experiencia misma ${ }^{28}$.

Por reacción positiva comprendemos, siguiendo a Kant, la atracción que se presta a ser mantenida; por reacción negativa, lo contrario — permitiendo la reacción mixta del horror fascinado. Más que el placer, lo característico de una reacción estética positiva sería, según Budd, que la experiencia resulte inherentemente gratificante ${ }^{29}$, debiendo cumplir con el carácter kantiano de ser «desinteresada» y no estar basada en la satisfacción de que el mundo sea de cierta manera ${ }^{30}$, pues ningún placer estético es proposicional.

Su definición menos conservadora tal vez sea la que estipula para la noción de «propiedades experimentadas»:

«aquellas propiedades que experimentamos que el item posee, según la percepción, el pensamiento o la imaginación, y la noción ha de ser entendida en un sentido plenamente abarcante, cubriendo no sólo las propiedades inmediatamente perceptibles, sino también las relacionales, representacionales, simbólicas y emocionales, tal y como estén dispuestas en el item, e incluyendo la clase o el tipo de cosa que sea el item y por la cual o el cual lo experimentamos» ${ }^{31}$.

En esta definición solamente da cobijo a la acepción reformada del «desinterés», aquel que traía consigo la ampliación del alcance de objetos disponibles y que, siguiendo su estímulo, da cabida a un rango mayor de productos de la experiencia, incluyendo no sólo a la actividad perceptiva, reproductora de impresiones, sino también a los diversos productos de la imaginación y el pensamiento —incluidos los conceptos. A la ampliación del alcance de objetos se le suma el alcance de los productos de la experiencia. El desarrollo de este planteamiento tendrá como resultado el crecimiento de las cuestiones estéticas que se le asocian (como, por ejemplo, la apreciación estética del deporte o de los actos de circo, entre otros).

Después de su análisis, Budd define la respuesta estética a la naturaleza, como naturaleza como la adición de las características, por un lado, de la concepción interna de la respuesta a la naturaleza y, por otro, las de la respuesta estética, en general. De modo que respondemos estéticamente a un item natural (del cuál podemos conocer su concepto, su clase o simplemente asociarlo al concepto de naturaleza en general) si al contemplarlo apreciamos sus propiedades estéticas como gratificantes o displacientes, de forma desin-

28 M. Budd, 2005, p. 14.

29 M. Budd, 2005, p. 15: «En una concepción del placer más fina que la de Kant, aunque una reacción positiva bien puede ser una experiencia de placer, no tendría por qué ser necesariamente así. Lo que es esencial a una reacción positiva es que el sujeto debe encontrar que la experiencia del objeto resulta, de forma inherente, gratificante.»

30 M. Budd, 2005, p. 14.

31 M. Budd, 2005, p. 14. 
teresada. Y serán cuatro los tipos de cualidades capaces de mostrarnos la diversidad de atractivos estéticos de la naturaleza, como naturaleza: las características de las distintas fases del crecimiento, las diversas formas de ítems y objetos, el movimiento de los seres vivos en relación y la adecuación de las partes y el todo de los seres vivos y el entorno.

Respecto a la cuestión concreta del protagonismo del concepto en la apreciación estética, Budd defiende que el hecho de que seamos capaces de identificar y clasificar objetos como pertenecientes a diversas clases o tipos, en base a su apariencia, nos brinda una conciencia más rica de la naturaleza en su conjunto, pero, a su juicio, ello no nos provee de una apreciación mejorada de la naturaleza, tal y como el cognitivismo pretende. No obstante, Budd admite que hay casos en los que cierta comprensión de la naturaleza del fenómeno transforma nuestra experiencia estética de la naturaleza. Es evidente que, en mayor o menor grado, todos disponemos de ciertas concepciones científicas de la naturaleza bajo las que agrupamos sus fenómenos y de las que a su vez obtenemos, en esa misma variedad de rango, distintos grados de comprensión. Es posible que tengamos conocimiento preciso de determinados procesos cosmológicos, pero que desconozcamos los detalles comprensivos que posee un ornitólogo. En ese caso, podremos asociar a nuestra experiencia ciertos pensamientos o emociones que resultan relevantes estéticamente y que, no estando disponibles para el neófito, realzan nuestra experiencia (si observamos, por ejemplo, el resplandor de un meteoro ardiendo en la atmosfera terrestre, al que popularmente llamamos «estrella fugaz»). Budd admite que la transformación de la experiencia estética obtenida a partir de ese conocimiento relevante, trae consigo la posibilidad ampliar la variedad de apreciación estética de la naturaleza y de sus respuestas, que de otro modo no estarían disponibles. Pero eso no implica que siempre aumente el deleite estético, ya que podría incluso cancelarlo (por ejemplo, al observar una planta que sabemos venenosa), ni que ese conocimiento específico sea responsable de la corrección estética de la apreciación.

Budd acepta que en ocasiones el conocimiento modifica y mejora la apreciación estética, ampliando la respuesta y sus tipos, pero advierte que no siempre sucede así. Hay casos en los que la información no modifica la experiencia estética, como por ejemplo, si al apreciar un árbol precisamos si se trata de un olmo o de un abedul. Y si por alguna razón percibimos equívocamente un item, como siendo de una clase a la que no pertenece, esa equivocación tendrá consecuencias cognitivas, pero no estéticas, puesto que no hay error en la percepción, sino solamente en la asignación del concepto de clase que le hemos atribuido. Por tanto, la apreciación estará mal fundada sólo en ese sentido, pero no será incorrecta, ni mucho menos la podremos tildar de falsa — como solicita Carlson para el contraejemplo ${ }^{32}$ de una apreciación mediada por algún tipo de interés.

Tampoco admite Budd la analogía de la estructura de la apreciación estética que Carlson traza entre arte y naturaleza. El rechazo de Budd tiene su base en los requerimientos fenomenológicos de la experiencia que considera distintos para arte y naturaleza y no asimilables. Abundando sobre esta cuestión, Budd propone dos pruebas de satisfacibilidad para esta afirmación: una en la que admitimos contemplar la naturaleza como arte; otra, en la que afirmamos que la apreciación estética de la naturaleza debe ser verdadera con la fenomenología de nuestra experiencia. Budd demuestra que no es posible satisfacer ambos

32 M. Budd, 2005, p. 23. 
extremos apoyándose en la concepción de la belleza de Anthony Savile. Aunque Savile asimila la apreciación estética de la naturaleza a la apreciación del arte, reconoce que la estética de la naturaleza posee un carácter distintivo: mayor libertad y relatividad. Para Savile, un juicio de algo bello es un juicio de ese algo bajo una descripción (un problema) y un estilo. Haciendo un uso similar al que Carlson hiciera con la deducción de las categorías de Walton, el recorrido argumental nos lleva a admitir, en primer lugar, que la posibilidad de observar un objeto natural como si fuera arte no implica ni necesidad, ni actualidad; por otro lado, vemos que cualquier forma de considerar la naturaleza como si fuera arte implicaría el ser conscientes de aplicar conceptos esenciales para el arte a la naturaleza, lo que cambiaría su fenomenología. Sin embargo, no es necesario que la experiencia estética de la naturaleza incluya esa conciencia de ser como arte. Las experiencias estéticas del arte y la naturaleza son fenomenológicamente distintas y así, por tanto, lo es el placer que obtenemos en cada una de ellas. La satisfacción estética que podemos experimentar al apreciar un objeto natural, como natural, añade a la ampliación de objetos, alcanzada con la reforma de la noción de desinterés, la ampliación del tipo de experiencias relevantes para la apreciación estética y, en consecuencia, da lugar a un aumento de los focos relevantes de deleite estético, correspondientes a cada uno de esos tipos de experiencia (emotiva, simbólica...), además de la enfocada por categorías científicas.

\section{La crítica de Budd a la estética positiva}

Budd levanta su crítica a las tesis de la estética positiva sobre lo que considera tres debilidades internas de la concepción. A su juicio, la estética positiva necesitaría definir mejor los extremos de su alcance, su fuerza y su estatus modal. En primer lugar, Budd apunta que debería especificarse si el alcance afecta a la naturaleza como un todo, a la biosfera terrestre o, por ejemplo, a cada ecosistema. En relación a su fuerza, habría que considerar la posibilidad de que, dentro del carácter positivo general de esta concepción, tuviera cabida la atribución de ciertas cualidades negativas a (de) los ítems, compensadas con las positivas, tal y como plantea Eugene Hargrove ${ }^{33}$. Por último, en relación a su estatus, Budd reflexiona sobre si cabe afirmar alguna verdad necesaria o no de la naturaleza.

El primer argumento que Budd critica es la deducción de la bondad estética positiva a partir de la deducción de categorías estéticas correctas, esto es, las científicas, a partir del modelo histórico artístico. Budd considera que con ello no queda demostrado que la naturaleza requiera ser juzgada estéticamente por las categorías aportadas por las diferentes ciencias ya que, según Carlson, la ciencia garantiza esa bondad en base a las cualidades estéticas que ella misma postula en la naturaleza: «orden, regularidad, armonía, equilibrio, tensión, conflicto...». Budd divide estas cualidades en dos grupos, calificando como negativas a «la tensión y el conflicto», mientras que reserva a «la resolución» la posibilidad de ser antiestética. Apelar a estas cualidades como forma de dar peso al argumento, por tanto, no alcanza para Budd su propósito. El segundo grupo de cualidades, orden, regularidad, armonía y equilibrio, no pasan de ser para Budd «pequeñas reflexiones del escasamente

33 E. Hargrove, Foundations of Environmental Ethics, Englewood Cliffs, NJ: Prentice Hall, 1989. 
regulado carácter de la naturaleza» ${ }^{34}$, del cual tampoco se sigue que todos los objetos sean equiparables en atractivo estético. De hecho, a pesar de la regulación científica en base a ciertas cualidades, los objetos varían de atractivo y manifiestan diferentes cualidades estéticas. A ello se añade que la regulación de un tipo de atractivo, no excluye la existencia de cualidades negativas, ni garantiza que un objeto que las posea, manifieste un balance positivo general que se decante a favor de sus cualidades positivas. Finalmente, Budd sostiene que un objeto cuyo balance oscile a favor de sus cualidades estéticas negativas, como en el caso de un objeto exageradamente malformado, nos resultará grotesco o estéticamente desagradable a pesar de que la ciencia sea capaz de explicar el origen de su malformación. A su juicio, desde el punto de vista de la apreciación de la naturaleza en general, existe un gran número de cuestiones sobre el origen y los procesos de la naturaleza a ser comprendidos científicamente, pero aún aceptando que sea así, esto deja sin tocar la cuestión de cuáles sean las cualidades estéticas de un item. El orden impuesto a un objeto por la interacción natural de procesos y fuerzas, junto con la explicación científica correspondiente, no implica, desde el punto de vista estético, que todo objeto tenga que poseer el mismo valor y atractivo estético. Para Budd, Carlson intercambia el valor semántico de dos expresiones, a saber: atribuye la característica de ser «igualmente apreciable» tanto a «evidenciar igualmente el orden natural» como a ser «equiparable en valor estético» ${ }^{35}$.

El segundo argumento de Carlson que Budd expone a crítica es el que explicita que la apreciación de la naturaleza ha de ser entendida como una forma de «apreciación de orden». Budd indica que «orden» para Carlson significa «patrón ordenado de forma no artística y no estética», el cual es resultado de la imposición de ciertas fuerzas azarosas o no, responsables de que resulte apreciable. En el caso de la naturaleza, el orden relevante sería el orden natural, las fuerzas relevantes las biológicas, geológicas y meteorológicas (productoras del orden), y la visión relevante la que ofrecen las distintas ciencias. Debido a que todo en la naturaleza participa de un modo u otro de ese orden, se da una equiparación del atractivo estético de todos los objetos naturales, de forma que seleccionar unos objetos frente a otros como más o menos bellos resultaría irrelevante. Este argumento no impide que lleguemos a la conclusión de que no todo objeto natural es igualmente bello, con independencia de en qué fase o estado vital se encuentre, ya esté malformado, desnutrido o enfermo, y a pesar de que esos estados sean científicamente explicables por la intervención de las fuerzas naturales que los producen, como partes de ese patrón. Para Budd, cualquier argumento que brinde esta conclusión es defectuoso y, a su juicio, los dos argumentos de Carlson la animan.

A pesar de su crítica a los argumentos de Carlson, Budd se cuestiona si es posible conservar alguna versión de la estética positiva, para lo cuál propone una modificación de su alcance. Budd propone que una versión más plausible sería una que formulara afirmaciones de dos tipos: o bien sobre clases, o bien sobre ecosistemas. Resulta más factible pensar que, quizá, todas las clases naturales estén dotadas de algún tipo de atractivo. A ello, cabría plantear dos dificultades o excepciones. La primera afectaría a las clases naturales, cuando no constituyan formas de vida y cuando, por su carácter, parezca inadecuado asegurar que poseen valor estético positivo, dejando abierta la posibilidad general de que exista alguna

34 M. Budd, 2005, p. 101

35 M. Budd, 2005, p. 102.

Daimon. Revista Internacional de Filosofía, $\mathrm{n}^{\circ}$ 62, 2014 
clase natural que no posea ese valor. $\mathrm{Si}$, en segundo lugar, aplicamos la afirmación a los ecosistemas y postulamos que probablemente cada uno posea valor estético positivo, se nos presentan tres cuestiones.

La primera es relativa a la propia afirmación: si es cierto que podemos atribuir belleza a todo ecosistema, sería necesario que ésta proviniese de su carácter de ecosistema. La segunda afecta al valor estético que postulamos y que aquí depende directamente de la definición de ecosistema. Al plantearnos la belleza como resultado de la interacción de fuerzas, medio y seres, sucede que el ecosistema desarrolla tanto procesos generadores de vida, como fases de ocaso, destrucción o muerte, con el consiguiente contenido expresivo de dolor o sufrimiento, no quedando claro cómo podría un ecosistema fundar un valor estético positivo en base a sus propias características. La tercera forma del planteamiento es para Budd la más plausible. En ella se nos invita a considerar a los ecosistemas como conjuntos biológicos creadores y regeneradores de la propia vida. En este enfoque podemos dar cabida a cuatro ideas: la que nos dice que la mayor parte de las formas vivas de un ecosistema son bellas en sí mismas; otra que nos muestra cómo la fealdad local aparece como punto de un proceso que se sucede por belleza, una tercera que nos muestra cómo esa fealdad disminuye al considerarla parte del preludio de una nueva vida; y, por último, que el sistema es un conjunto de procesos bellos que también resulta bello en sí mismo. Budd se inclina por que la belleza que postulamos es soportada argumentalmente o bien por la primera idea, o bien por la cuarta.

Aún así, si quisiéramos dar por válido este argumento no tendríamos más remedio que admitir ulteriores límites espacio-temporales mayores a los que nos ofrece la duración vital del ecosistema, ya que el concepto de naturaleza en general los incluye. Otro problema viene dado, advierte Budd, por la evidencia de que cada uno de nosotros apenas podemos apreciar una pequeña parte de cada ecosistema, no prestándose nunca, en ningún caso, a una apreciación de conjunto ni en función de su espacio, de su duración, ni de sus elementos individuales o interactuando. Este problema no puede evitarse apelando a la relevancia del conocimiento científico o ecológico y es correlato de la inaccesibilidad estética de la naturaleza planteada por Kant. Así, al igual que la lectura de una novela, como obra temporal, implica recorrerla siguiendo el camino propuesto por su narración, del mismo modo apreciar un ecosistema, como objeto temporal, exigiría de nosotros una acción similar. «Pero diez mil sucesos esenciales en la estabilidad de un ecosistema tienen lugar en él de esa manera ${ }^{36}$, en ocasiones, menciona Budd, bajo tierra y más allá de los límites de nuestra observación. Por tanto, la idea de que un ecosistema posee valor estético positivo nada implica acerca del valor de sus ítems particulares. Considerados por sí mismos, no podemos pensar que siempre tengan valor positivo, pero esta idea varía si lo asociamos a la belleza del ecosistema como todo.

Para Budd, fuera de esta concepción reconsiderada de la estética positiva, nada en su idea de apreciación estética de la naturaleza, como naturaleza, exige que para apreciar un item necesariamente tengamos que valorar su función y parte en un ecosistema. Pero, si desde sus filas reclamásemos el concepto de ecosistema para atribuirle a este enfoque un alcance desde el que fundar la afirmación de que toda la naturaleza virgen es bella, deberíamos entonces hacernos responsables de que la misma noción exige de nosotros que juzguemos ese item con todas las consecuencias, como parte y función del mismo.

36 M. Budd, 2005, p. 105 


\section{Conclusión}

La concepción de Malcom Budd acerca de la apreciación estética del mundo natural es, desde el principio, más abarcante y completa que la defendida por Allen Carlson. En primer lugar, porque diferencia entre apreciar la naturaleza sin necesidad de atender exactamente a lo que esta es (definida en contraposición a lo que no es: arte/artefacto), tomándola como objeto estético a cuyos diversos estímulos podemos responder sin restricción cognitiva alguna, y apreciar la naturaleza, como naturaleza, por lo que esta es. También esta segunda vía cuenta con una concepción más generosa que la planteada por Carlson ya que, los requisitos conceptuales que en ella se comprenden incluyen desde el caso más indeterminado, aquel en el que, ante la ausencia de un concepto, la apreciación esté mediada por el concepto de naturaleza en general (atendiendo a los inequívocos rasgos fenomenológicos que ostentan los items naturales), pasando por los casos intermedios, en los que la apreciación está guiada por conceptos no científicos de diverso origen, hasta llegar al caso más determinado, en el que la apreciación esté mediada por conceptos científicos. La concurrencia de un concepto de este tipo no implicaría para Budd, como para Carlson, que facilite, colabore, realce o incremente nuestra experiencia estética, ya que hay casos en los que la información entorpece, reduce o imposibilita el deleite estético, no siendo, por ello, imprescindible. La mediación de los conceptos científicos en la experiencia estética de la naturaleza es para Budd apenas uno de los casos posibles de la apreciación mediada por conceptos. Siendo así, la primera ventaja de la concepción de Budd frente a la de Carlson es que no restringe las posibilidades de apreciación estética de la naturaleza, en dependencia de otro concepto que no sea el de naturaleza, en general. La segunda, que tampoco restringe la apreciación según los distintos usos de la idea de desinterés y la subsiguiente exigencia o no de distancia estética. Uno puede estar en un paraje y adoptar una posición distante, buscando el gozo formal de una escena, contemplada según una posición estética tradicional, al tiempo que en otra ocasión podrá comprometerse estéticamente con su entorno, dejándose llevar por sus múltiples estímulos sensoriales y por la excitante diversidad que provoca la carencia de marco estético. Por ello, la admisión del concepto renovado del desinterés no supone, en Budd, la denostación de la noción tradicional, la cual permanece vigente para sus antiguas aplicaciones, que no se cancelan.

Si atendemos al caso concreto en el que apreciamos la naturaleza según su origen natural, esto es, como naturaleza, debemos comprometernos en primer lugar con una definición general de qué es naturaleza y con su alcance. Tanto en Carlson como en Budd, la apreciación de la naturaleza se opone a la del arte y a la de los objetos producidos como artefactos, debido a su origen intencional. Sin embargo, es importante señalar que para Budd no todas las actividades y/o productos fabricados por el hombre caen dentro de la categoría de artefactos pues, al igual que los animales construyen nidos, colmenas, diques, madrigueras, etc., en su entorno, con materiales naturales, también el hombre, como ser natural, desarrolla actividades y productos del mismo tipo (caminos, pastoreo, hornos, barcas, puentes...) que podemos apreciar como naturaleza. La matización de Budd no es inocente, pues al aceptarla compromete severamente los términos en los que un entorno puede o no ser considerado como naturaleza virgen. Con ello se modificaría el alcance de la tesis de la estética positiva, la cuál se dirige, al menos en la formulación de Carlson, a la naturaleza «intocada», «no intervenida», en ningún sentido por la 
acción o la presencia del hombre en el entorno natural. Con su defensa del hombre como actor/ productor natural en el entorno, Budd deja una puerta abierta a posibles tipos de convivencia armónica del hombre en el entorno natural, si bien sería necesario definir de forma precisa cuáles son los rasgos de esos productos o los tipos de intervenciones que caerían dentro de ese grupo. Asimismo, a diferencia de Carlson, Budd incide, al igual que Kant, en lo que considera requisito fenomenológico implícito en toda experiencia estética de la naturaleza, por medio del cuál resulta innecesaria la deducción de categorías correctas propuesta por Carlson para distinguir los casos de objetos artificiales de apariencia natural. La apreciación estética de la naturaleza y sus objetos se muestra incompatible para fenomenologías distintas, tal y como ya explicara Kant por medio de su conocido ejemplo del falso canto de pájaro. ${ }^{37}$

Finalmente, la defensa del conocimiento científico como requisito para la apreciación estética de la naturaleza expuesta por Carlson adolece, en mi opinión, de una fundamentación concluyente. En primer lugar, porque en la tesis principal, la ciencia ocupa el lugar y el valor del conocimiento histórico, en paralelo con el que le es atribuido a la historia y a la crítica del arte, para la apreciación de obras de arte. Sin embargo, al mismo tiempo, Carlson busca después establecer una deducción de categorias comunes necesarias para la ciencia y la estética según las cuáles poder exigir el régimen cognitivo que le imprime al juicio estético y que defiende a través de las tesis de la estética positiva, como vimos: orden, regularidad, armonía, equilibrio, etc. El tránsito de uno a otro punto es el desarrollado por medio de la deducción de categorías de Walton, argumento débil que es eficazmente atacado por la contraargumentación de Budd. Finalmente, y a pesar de que Carlson asuma con Kant la finalidad formal de la naturaleza como principio de todos nuestros juicios, la tesis de Carlson resulta también vulnerable frente al marco general de la Crítica del Juicio, establecido según la idea de naturaleza como objeto científicamente cognoscible, pero estéticamente inaccesible, debido a que su enorme diversidad desborda la experiencia que somos capaces de tener de ella, impidiéndonos formarnos un sistema deductivo general, según principios, de las categorías de sus productos.

\section{Bibliografía}

BERLEANT, A., The Aesthetics of Environment, Philadelphia: Temple University Press, 1992.

BRADY, E., Aesthetics of the Natural Environment, Edinburgh University Press, 2003.

BUDD, M., The Aesthetic Appretiation of Nature, Oxford, Oxford University Press, 2005.

CARLSON, A., Asthetics and The Environment. The Appretiation of Nature, Art, and Architecture, New York, Routledge, 2000.

CARROLL, N., «On Being Moved by Nature: Between Religion and Natural History», en: Beyond Aesthetics, Cambridge University Press, 2001.

GOLDVLOVITCH, S., «Evaluating Nature Aesthetically», Journal of Aesthetics and Art Criticism, 56 (2), 1998, p.113-125.

HARGROVE, E., Foundations of Environmental Ethics, Englewood Cliffs, NJ: Prentice Hall, 1989.

37 I. Kant, Crítica del Juicio, $\S 42$. 
HEYD, TH., Encountering Nature. Toward an Environmental Culture, Aldershot, Ashgate, 2007.

HEPBURN, R., «Contemporary Aesthetics and the Neglect of Natural Beauty», en: A. Berlant y A. Carlson, (eds): The Aesthetic Appretiation of Natural Environments., Canada, Broadview Press, 2004.

KANT, I., Crítica del Juicio, Madrid, Austral, 1981.

SAVILE, A., The test of time: an essay in philosophical aesthetics, Clarendon Press, 1982. 
